\title{
TERRASAR-X / TANDEM-X FORMATION CONTROL - FIRST RESULTS FROM COMMISSIONING AND ROUTINE OPERATIONS
}

\author{
Ralph Kahle (1), Benjamin Schlepp (2), Michael Kirschner (3) \\ (1) German Aerospace Center (DLR), Münchner Str. 20, 82234 Wessling, Germany, \\ +49-8153-282451, Ralph.Kahle@dlr.de \\ (2) German Aerospace Center (DLR), Münchner Str. 20, 82234 Wessling, Germany, \\ +49-8153-282124, Benjamin.Schlepp@dlr.de \\ (3) German Aerospace Center (DLR), Münchner Str. 20, 82234 Wessling, Germany, \\ +49-8153-281385, Michael.Kirschner@dlr.de
}

\begin{abstract}
This paper describes the TerraSAR-X / TanDEM-X formation control concept, which bases on the relative eccentricity / inclination vector separation method, implemented within GSOC's flight dynamics system. The paper specifically elaborates on the results obtained during the first six month of the formation flying mission showing the safe and precise automated groundcontrol of the space-borne radar interferometer.
\end{abstract}

Keywords: TerraSAR-X, TanDEM-X, formation flying, flight dynamics operations

\section{Introduction}

The primary goal of the TerraSAR-X mission (TSX, launched on 15 June 2007) is the provision of high-resolution Synthetic Aperture Radar (SAR) data to both science and commercial users. At 21 June 2010 an almost identical satellite, TanDEM-X (TDX), was launched in order to form the first configurable SAR interferometer employing formation flying with TSX. The main objective of the common TanDEM-X (TerraSAR-X add-on for Digital Elevation Measurement) mission is to generate a global digital elevation model (DEM) with unprecedented accuracy as the basis for a wide range of scientific research as well as for commercial DEM production [8]. After successful completion of the mono-static and bi-static radar instrument commissioning phases the two satellites started the routine operation as a large single-pass SAR interferometer in December 2010. In order to collect sufficient measurements for a global DEM, three years of formation flying are foreseen with flexible baselines ranging from $200 \mathrm{~m}$ to few kilometers. Table 1 summarizes the TanDEM-X mission timeline.

Table 1. TanDEM-X mission timeline

\begin{tabular}{ll}
\hline Phase & Epoch \\
\hline Launch & $2010-06-21$ \\
\hline Drift phase to acquire target orbit & $2010-06-22$ to 07-22 \\
\hline Wide formation: TDX mono-static radar instrument commissioning & $2010-07-22$ to 10-12 \\
\hline Reconfiguration & $2010-10-12$ to 10-15 \\
\hline $\begin{array}{l}\text { Close formation: TSX/TDX bi-static instrument commissioning; Begin of } \\
\text { routine mono-static radar operation on both satellites }\end{array}$ & $2010-10-15$ to 12-12 \\
\hline Begin of routine DEM acquisition with flexible baselines & $2010-12-12$ \\
\hline
\end{tabular}

DLR is responsible for the scientific exploitation of the TanDEM-X data as well as for planning and implementing the mission, controlling the two satellites and generating the digital elevation model. Astrium built the satellites and shares in the cost of its development and exploitation. As with TerraSAR-X, the responsibility for marketing the TanDEM-X data commercially lies in the hands of Infoterra GmbH, a subsidiary of Astrium. 
The satellites were built by Astrium GmbH. The TanDEM-X satellite follows the TerraSAR-X design with minor modifications such as an additional cold gas propulsion system (powered by high-pressure nitrogen gas) to enable fine-tuning of its relative position during formation flying and an additional S-band receiver to receive status and position information sent by TerraSAR-X. This data is utilized within TanDEM-X Autonomous Formation Flying System (TAFF) for purpose of relative navigation and optionally closed loop in-plane formation maintenance. The TanDEM-X satellite has been designed for a nominal lifetime of five years and has a planned overlap with TerraSAR-X of three years. TerraSAR-X holds consumables and resources for up to seven years of operation however, potentially allowing for a prolongation of the overlap and the duration of the TanDEM-X mission.

Table 2. TerraSAR-X / TanDEM-X satellite characteristics (FD-relevant parameters only)

\begin{tabular}{|c|c|c|}
\hline & TerraSAR-X & TanDEM-X \\
\hline Launch date & 2007-06-15 & 2010-06-21 \\
\hline Dimension & \multicolumn{2}{|c|}{ Height: 5 meters, Diameter: 2.4 meters } \\
\hline Mass (2010-11-09) & $1325 \mathrm{~kg}$ & $1341 \mathrm{~kg}$ \\
\hline Propulsion system & $\begin{array}{l}4 \times 1 \mathrm{~N} \text { thrusters in anti-flight } \\
\text { direction - } 78 \mathrm{~kg} \text { Hydrazine }\end{array}$ & $\begin{array}{l}4 \times 1 \mathrm{~N} \text { thrusters in anti-flight } \\
\text { direction }-78 \mathrm{~kg} \text { Hydrazine, } \\
2 \times 40 \mathrm{mN} \text { thrusters in each } \\
\text { flight and anti-flight direction } \\
-36 \mathrm{~kg} \text { Cold-Gas (Nitrogen) }\end{array}$ \\
\hline GPS receivers & \multicolumn{2}{|c|}{$\begin{array}{l}\text { MosaicGNSS single-frequency (EADS/Astrium), } \\
\text { IGOR dual-frequency (BRE/JPL/GFZ Potsdam) }\end{array}$} \\
\hline Inter-satellite communication & S-Band transmitter & S-Band receiver \\
\hline other & & $\begin{array}{l}\text { TanDEM-X Autonomous } \\
\text { Formation Flying System } \\
\text { (TAFF) }\end{array}$ \\
\hline
\end{tabular}

The first section of the paper gives an overview on the guidance, navigation and control approach implemented for formation monitoring and control within the Flight Dynamics System (FDS) at German Space Operations Center (GSOC), where the complete FD system design, development and implementation was performed. The second part of the paper is devoted to the results obtained within the commissioning phases (CP) and beginning of routine DEM acquisition in December 2010.

\section{Formation Control Concept}

The TDX/TSX relative orbit control concept is based on the relative eccentricity / inclination vector separation method. For a detailed description please refer to [4] and [5] and the references cited therein. In the frame of this paper only a short summary is given since the main focus is on the flight results.

The relative orbital elements parameterization used in this paper is based on the relative semi-major axis $\Delta a$, the relative eccentricity vector $a\left(\Delta e_{x}, \Delta e_{y}\right)^{T}$, the relative inclination vector $a\left(\Delta i_{x}, \Delta i_{y}\right)^{T}$, and the relative mean argument of latitude $a \Delta u$. The six relative elements are derived from combinations of the mean Keplerian elements of TSX and TDX as follows: 


$$
\left(\begin{array}{c}
\Delta a \\
a \Delta e_{x} \\
a \Delta e_{y} \\
a \Delta i_{x} \\
a \Delta i_{y} \\
a \Delta u
\end{array}\right)=\left(\begin{array}{c}
a_{T D X}-a_{T S X} \\
a_{T S X}\left(e_{T D X} \cos \omega_{T D X}-e_{T S X} \cos \omega_{T S X}\right) \\
a_{T S X}\left(e_{T D X} \sin \omega_{T D X}-e_{T S X} \sin \omega_{T S X}\right) \\
a_{T S x}\left(i_{T D X}-i_{T S X}\right) \\
a_{T S X}\left(\Omega_{T D X}-\Omega_{T S X}\right) \sin i_{T S X} \\
a_{T S X}\left(u_{T D X}-u_{T S X}\right)
\end{array}\right)
$$

with the Keplerian elements semi-major axis $a$, eccentricity $e$, argument of perigee $\omega$, inclination $i$ and right ascension of the ascending node $\Omega$. The mean argument of latitude $u$ is the sum of argument of perigee and mean anomaly, i.e. $u=\omega+M$.

\subsection{Target Formation Geometry}

The starting point for operating the TSX-TDX formation flying mission is the TanDEM-X acquisition plan (TAP), which contains a timeline of formation geometries and bi-static SAR acquisitions needed to generate a global DEM within a period of 3 years. The process of optimization of the data acquisition plan and constraints are described in [8]. The TAP target formation parameters together with allowed tolerances are provided to flight dynamics, where they are converted into the relative orbital elements. Because of the advantageous geometrical meaning (e.g. when picturing the formation evolution or defining formation control dead-bands), the polar representation of the relative eccentricity and inclination vectors is used:

$$
\Delta \vec{\alpha}_{\text {target }}=\left(\begin{array}{llllll}
\Delta a & a \delta e & \varphi & a \delta i & \theta & a \Delta u
\end{array}\right)^{T}
$$

with the magnitude of the relative eccentricity vector $a \delta e$, the relative argument of perigee $\varphi$, the magnitude of the relative inclination vector $a \delta i$, and the relative ascending node $\theta$. The polar and Cartesian representations (as used in Eq. 1) are transferred by

$$
\Delta \vec{e}=\left(\begin{array}{c}
\Delta e_{x} \\
\Delta e_{y}
\end{array}\right)=\delta e\left(\begin{array}{c}
\cos \varphi \\
\sin \varphi
\end{array}\right) \text { and } \Delta \vec{i}=\left(\begin{array}{c}
\Delta i_{x} \\
\Delta i_{y}
\end{array}\right)=\delta i\left(\begin{array}{c}
\cos \theta \\
\sin \theta
\end{array}\right) \text {. }
$$

Typically, the TAP sampling interval is 11 days according to the TSX repeat cycle. In case of more frequent formation geometry changes, intermediate sampling is possible. A linear interpolation of the target relative elements is applied in-between the sample points.

Fig. 1 depicts the change of horizontal and vertical separation ( $a \delta e, a \delta i)$ over the period of the bistatic commissioning phase (October 18 to December 10, 2010) and the beginning of the routine DEM acquisition. While the formation geometry was kept constant over the commissioning phase, regular changes were typically performed at the borders of the 11-days cycle during the routine phase. So far the other target formation parameters have been kept constant, i.e. $\varphi=260$ deg, $\theta=$ $90 \mathrm{deg}$, and relative along-track distance $\Delta T=0 \mathrm{~m}$. Because of its dependency from horizontal separation, the target relative mean argument of latitude is not constant and can be determined as:

$$
a \Delta u=\Delta T-a \delta i \frac{\sin \theta}{\tan i_{T S X}} .
$$




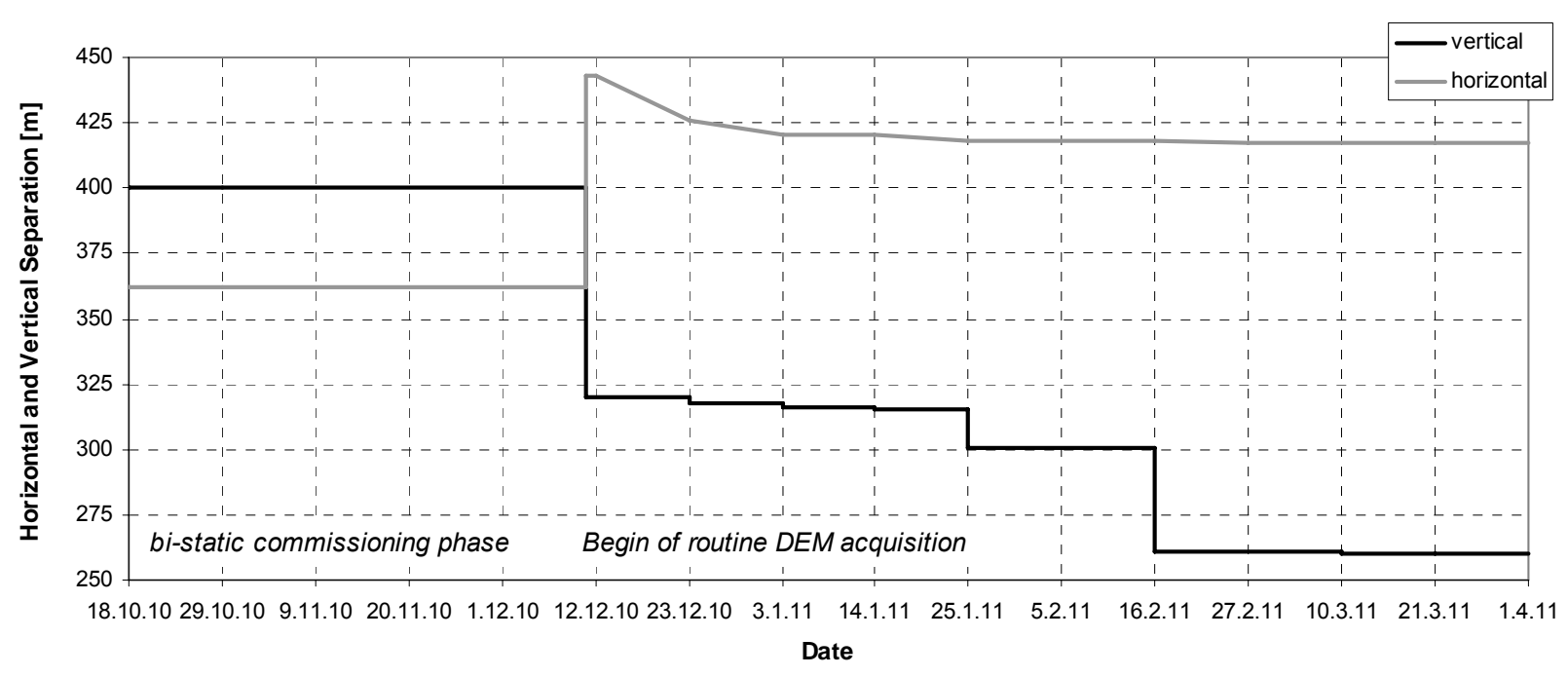

Figure 1. Target TDX-TSX separation in horizontal (a $\delta)$ and vertical (a $\delta$ ) direction.

Not shown in Figure 1 is the geometry used during the mono-static commission phase. Here TDX was $20 \mathrm{~km}$ behind of TSX. To achieve a similar ground-track as TSX, the horizontal separation was set to $1305 \mathrm{~m}$ which corresponds to the equator surface motion within $2.6 \mathrm{sec}$ of flight-time (or 20 $\mathrm{km})$. An anti-parallel relative eccentricity / inclination vector separation was set up with $a \delta e=300$ $\mathrm{m}, \varphi=270 \mathrm{deg}$ and $\theta=90 \mathrm{deg}$.

It has to be mentioned that the TAP formation timeline is not frozen for the 3 years of formation flight. For example, if the DEM acquisitions within a period of constrained formation geometry is unsuccessful (e.g. because of conflicts with TerraSAR-X mission or other reason) the formation reconfiguration has to be delayed in order to allow for re-acquisition of the particular data-takes. It is also likely that based on the experience gained from the first cycles of routine DEM acquisition the TAP process might be modified to improve the overall performance.

\subsection{Relative Navigation}

The IGOR dual-frequency GPS receiver (or single-frequency MosaicGNSS receiver as backup, cf. Tab. 2) telemetry data is pre-processed to extract GPS navigation data for use in orbit determination (OD) and raw data for precise orbit determination (POD). The OD performs a least-squares batch adjustment of the following estimation parameters: epoch state vector (position and velocity), drag coefficient, solar radiation coefficient, extended maneuvers, and measurement biases. The achieved orbit determination accuracy is about 3m (3D, RMS, cf. sect. 3.1). The POD is performed based on GPS carrier phase and pseudo-range data. Auxiliary data such as the GPS orbit and clock products, Earth orientation parameters, and S/C attitude information are acquired prior to the generation of the precise orbits. Here, the achieved accuracy is $5-10 \mathrm{~cm}$ (3D, RMS) depending on auxiliary data types [9].

For the purpose of relative navigation (RelNav) within the FD formation control system, the filtering of navigation solution data is preferred to the processing of raw pseudorange and/or carrier phase data. In that way the amount of auxiliary information is significantly reduced leading to higher robustness with sufficient relative orbit determination accuracy as will be shown later. A more sophisticated processing utilizes the raw measurements for precise baseline determination, which is essential for processing the bi-static SAR acquisitions [8]. Here relative orbit accuracies of 1-2 mm (3D, RMS) are achieved [9] providing a very precise reference for validation of the RelNav solution used in formation control system.

RelNav is basically the synchronous orbit determination of equal TSX and TDX navigation data arcs (i.e. typically 24 hours) using the same environment model parameters. As a result, common 
errors that are mainly related to atmospheric drag modeling are cancelled. The achieved relative position accuracy is assessed in section 3.1. The RelNav solutions are stored as satellite-specific state vector records and are used as input to the formation monitoring and control system. From the propagation of these state vectors osculating Keplerian elements are found, which are first converted into mean elements before building the relative orbital elements as described in Eq. 1. For monitoring purpose the relative orbital elements are also expressed in the Hill orbital frame $(\Delta R, \Delta T, \Delta N)$, which originates in the TSX Radial-Tangential-Normal (R-T-N) coordinate system. The corresponding distances in the three axes can be determined from a set of relative orbital elements as [4]:

$$
\left(\begin{array}{c}
\Delta R \\
\Delta T \\
\Delta N
\end{array}\right)=\left[\begin{array}{ccc}
\Delta a & -a \Delta e_{x} & -a \Delta e_{y} \\
a \Delta u & -2 a \Delta e_{y} & 2 a \Delta e_{x} \\
0 & -a \Delta i_{y} & a \Delta i_{x}
\end{array}\right] \times\left(\begin{array}{c}
1 \\
\cos u \\
\sin u
\end{array}\right) .
$$

The corresponding cross-track distance is:

$$
\Delta C=\sqrt{\Delta R^{2}+\Delta N^{2}} .
$$

\subsection{Control Concept}

Because of the fact that both scientific and commercial users rely on the provision of highresolution SAR images, a key requirement was defined to ensure that the original TerraSAR-X mission is not affected by the TanDEM-X mission. The orbit control concept therefore comprises of two steps: absolute and relative orbit control. Absolute orbit refers to the TSX reference trajectory which exactly closes after an 11-days repeat cycle. The TSX satellite is controlled to stay within a tube of 250 m radius around a predefined Earth-fixed reference orbit that enables highly repeatable data-take conditions. Orbit keeping maneuvers are conducted on semi-regular basis to adjust the TSX orbit to the reference trajectory. As illustrated in Fig. 2 for locations near the ascending node, the orbit raising maneuvers are conducted near the eastern (right-hand) boundary of the tube and induce a drift of the normal component in a western direction. This drift is ultimately reverted by atmospheric drag after which the satellite returns to the right-hand side (for details refer to [2]). Within the solar minimum period 2008-2009 in-plane control maneuvers with typically $1 \mathrm{~cm} / \mathrm{s} \Delta \mathrm{v}$ were performed every 10 to 14 days. Currently (Feb. 2011) a moderate increase in solar activity causes slightly higher drag and hence shorter maneuver cycle (about 1 week) and larger size (1.5 $2.0 \mathrm{~cm} / \mathrm{s}$ ). To counteract luni-solar perturbations on the inclination, out-of-plane maneuvers are performed 3-4 times a year with up to $30 \mathrm{~cm} / \mathrm{s} \Delta \mathrm{v}$.

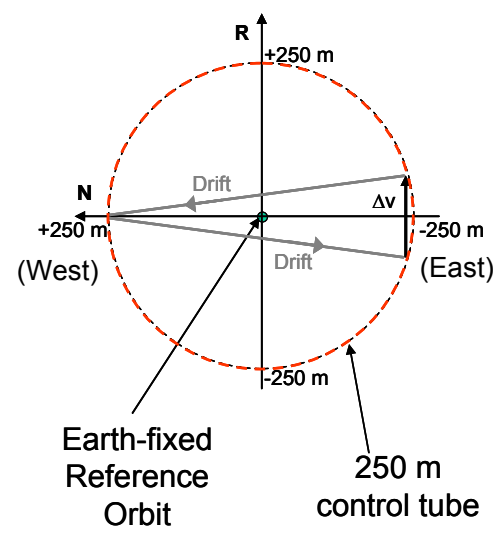

Figure 2. Schematic view of TSX motion relative to the predefined reference orbit at ascending node transits (R-T-N convention). 
The TDX/TSX relative orbit control concept is based on the relative eccentricity / inclination vector separation method. In order to meet the relative control requirements (i.e. $20 \mathrm{~m}$ cross-track (2D), and $200 \mathrm{~m}$ parallel to flight-direction) required for SAR interferometry TDX must both replicate the TSX orbit keeping maneuvers and compensate the natural deviation of the relative eccentricity/inclination vectors. While the $\mathrm{cm} / \mathrm{s}$-level absolute orbit maintenance is performed with four 1 Newton hydrazine thrusters, two 40 milli-Newton cold-gas thrusters are used exclusively onboard TDX (cf. Tab. 2) to control the formation including counterbalancing of possible along-track drifts resulting from differential hydrazine maneuver execution errors. Precautions are implemented to ensure that the failure of any TSX or TDX maneuver can not result in a relative geometry with less than 150 m cross-track distance (cf. [6]).

Due to the Earth oblateness, the relative eccentricity and inclination vectors are subject to secular perturbations. In the latter case, a drift of the relative inclination vector in the y-direction occurs, which is proportional to the inclination difference of the two satellites (i.e. $a \Delta i_{x}$, cf. Eq. 1). When choosing identical inclinations, the TSX and TDX right ascension of ascending node $\Omega$ rotate at the same angular velocity yielding stable horizontal baselines as used during the commissioning phases. However, for the DEM acquisition phase the horizontal baseline has to be adjusted frequently (cf. Fig. 1). Here, a small inclination offset in the TDX orbit is used to build up large horizontal baselines over time without the need for expensive $\Omega$-adjustment maneuvers. In order to install a certain horizontal baseline drift rate $a \Delta \dot{\Omega}$ the necessary inclination offset is determined as:

$$
a \delta \Delta i_{x}=\frac{a \Delta \dot{\Omega}}{\dot{\Omega}_{T S X} \tan i_{T S X}}
$$

with $\dot{\Omega}_{\text {TSX }}$ being the nominal sun-synchronous $\Omega$-drift rate.

On the other hand, the relative eccentricity vector reflects the secular perigee variation of the individual satellites. It performs a rotation about the origin of the relative eccentricity vector plane with a period of roughly 100 days. This drift needs to be compensated by suitable formation keeping maneuvers to maintain a stable configuration. The required daily along-track velocity increment is proportional to the relative eccentricity vector magnitude $a \delta e$ and thus the desired peak separation of the orbits in radial direction. For example, a $300 \mathrm{~m}$ vertical separation demands every day two burns of approx. $0.5 \mathrm{~cm} / \mathrm{s}$ each and separated by half a revolution [4]. These maneuvers are additionally used to adjust the along-track separation and to compensate possible differential drag effects. In order to reduce the total maneuver size and improve along-track control performance at the same time, the number of drift orbits in-between the maneuver pair has been introduced as a further variable in the maneuver planning process.

Within the control process, which automatically runs twice a day, the TDX-TSX relative motion is predicted over the period of the upcoming maneuver slot that depends on ground-station and SAR instrument constraints (cf. [6]). If a control window becomes violated, TDX formation control maneuvers are planned. In particular in-plane maneuvers are planned as response to violation of the target relative elements $a \delta e_{\min / \max }, \varphi_{\min / \max }$ and $a \Delta u_{\min / \max }$. Because of its natural drift, the relative argument of perigee $\varphi$ typically triggers the in-plane control, while the other two parameters become relevant in case of target parameter changes. For example, in Fig. 3 (middle) the natural drift of the relative eccentricity vector causes daily violations of the left control limit. Because of maneuver planning constraints these limits are sometimes slightly exceeded, which is uncritical to the control performance as will be discussed in sect. 3.2. The in-plane maneuver pair transfers the relative argument of perigee to the opposite (i.e. right) limit of the control window (cf. Fig. 7 for more detailed view on time-evolution of $\varphi$ ). In-between these two in-plane maneuvers the relative argument of latitude is adjusted, too, making use of the temporal offset in the relative semi-major axis $\Delta a$. Since the third control parameter $a \delta \Delta u$ usually can not be adjusted exactly (this would 
require a three-maneuver approach instead) a small a $\Delta u$-drift for the time after the 2nd maneuver is installed by setting a non-zero $\Delta a$. In that way the average along-track error over one relative control cycle ( 24 hours) is about zero.
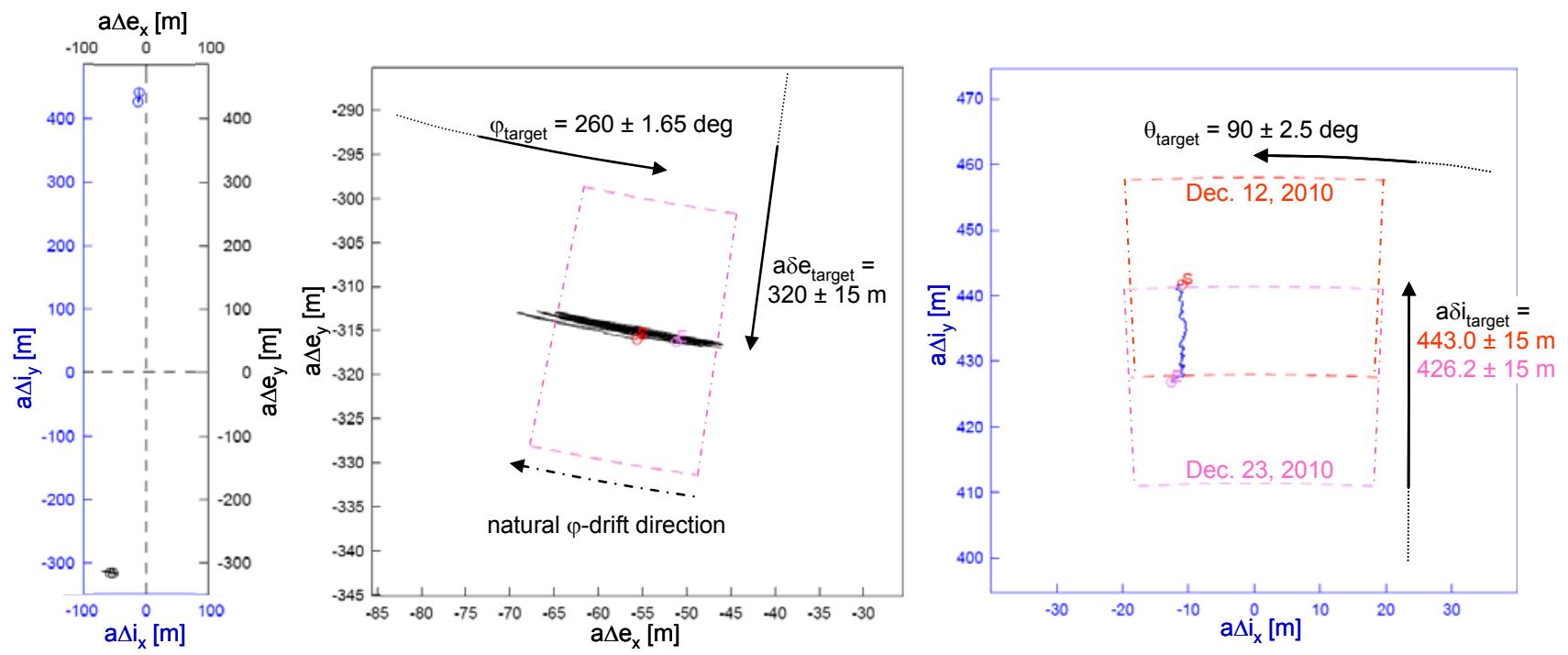

Figure 3. Relative eccentricity (middle) / relative inclination (right) vectors and control windows for the period Dec. 12-23, 2010. Left: total vector space.

The less frequent out-of-plane maneuvers are planned as response to violations of $a \delta i_{\min / m a x}$ and $\theta_{\min / \max }$ which usually appears only in case of target formation geometry changes. The right diagram of Fig. 3 depicts the sliding control window of the relative inclination vector for the interval from Dec. 12 to 23, 2010. At that time a large drift in the horizontal separation was installed, which afterwards became step-wise reduced and is currently stopped (cf. Fig. 1).

The formation control maneuvers direction and size are determined from desired changes in relative orbital elements by applying the simplified Gauss’ equations [4]:

$$
\left(\begin{array}{c}
\delta \Delta a \\
a \delta \Delta e_{x} \\
a \delta \Delta e_{y} \\
a \delta \Delta i_{x} \\
a \delta \Delta i_{y} \\
a \delta \Delta u
\end{array}\right)=\frac{1}{n}\left[\begin{array}{ccc}
0 & 2 & 0 \\
\sin u & 2 \cos u & 0 \\
-\cos u & 2 \sin u & 0 \\
0 & 0 & \cos u \\
0 & 0 & \sin u \\
-2 & -3 n\left(t-t_{\Delta v}\right) & -\sin u / \tan i
\end{array}\right]\left(\begin{array}{l}
\Delta v_{R} \\
\Delta v_{T} \\
\Delta v_{N}
\end{array}\right)
$$

Because of its lower efficiency and high operational effort radial maneuvers $\Delta v_{R}$ are not performed.

\section{Flight Results}

This section discusses the results obtained within the mono-static / bi-static instrument commissioning phases and at beginning of routine DEM acquisition. Because of the successful performance of an extensive ten-month formation control software simulation [6], most of the software fine-adjustments were done and the ground processing parameters were almost finally configured before TDX launch. Thus the commissioning of the formation maintenance function at end of July 2010 went very smooth and fully validated the control and operations concept. 


\subsection{Relative Navigation}

The (relative) orbit determination process is dump-triggered and therefore automatically runs up to 10 times a day. The accuracy of single satellite orbits is assessed by comparison to the precise orbits and is in general at the $3 \mathrm{~m}$ level (3D, RMS). The TDX-TSX relative navigation is validated from the comparison w.r.t. the precise baseline reconstruction results. Because of the cm-accurate POD, the comparison with mm-accurate baselines gives very similar results as obtained from differencing the POD-based absolute orbit accuracies. Typically the relative navigation as used in the formation control process has a cross-track (2D) accuracy $<0.5 \mathrm{~m}$ (RMS) and the along-track accuracy is on the $1 \mathrm{~m}$ level, which is sufficient for the purpose of formation monitoring and control with specified $20 \mathrm{~m}$ and $200 \mathrm{~m}$ control accuracies in cross-track and along-track directions, respectively .The accuracy assessment performed for Jan. 20, 2011 is summarized in Tab. 3 and furthermore illustrated in Fig. 4.

Table 3. Absolute and relative orbit determination accuracies on Jan. 20, 2011.

\begin{tabular}{|l|c|c|c|c|c|c|c|c|c|}
\hline \multirow{2}{*}[\mathrm{m}]{} & \multicolumn{3}{|c|}{ TS $\boldsymbol{X}$ OD } & \multicolumn{3}{c|}{ TDX OD } & \multicolumn{3}{c|}{ TD $\boldsymbol{X}$-TS $\boldsymbol{X}$ ReINav } \\
\cline { 2 - 11 } & Mean & STD & RMS & Mean & STD & RMS & Mean & STD & RMS \\
\hline Radial & -0.020 & 0.178 & 0.179 & -0.002 & 0.268 & 0.268 & 0.017 & 0.214 & 0.215 \\
\hline Along-Track & 2.245 & 0.958 & 2.441 & 1.329 & 0.905 & 1.607 & 0.908 & 0.631 & 1.106 \\
\hline Normal & 0.031 & 1.434 & 1.434 & 0.026 & 1.358 & 1.359 & -0.006 & 0.366 & 0.366 \\
\hline Total (3D) & 2.692 & 0.895 & 2.837 & 1.958 & 0.816 & 2.122 & 1.085 & 0.475 & 1.185 \\
\hline Cross-Track (2D) & 0.037 & 1.445 & 1.445 & 0.026 & 1.385 & 1.385 & 0.018 & 0.425 & 0.425 \\
\hline
\end{tabular}
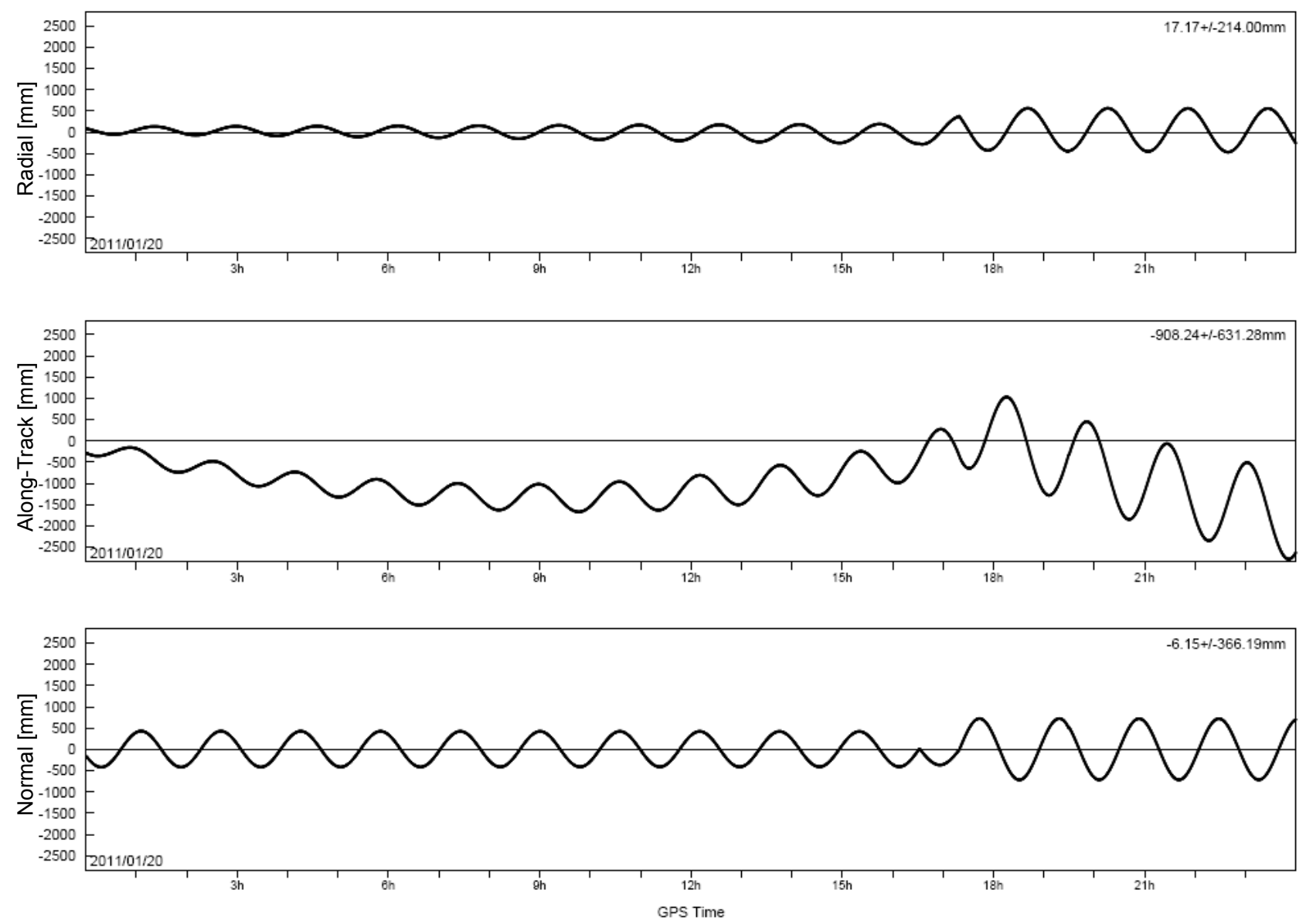

Figure 4. Relative navigation accuracy [mm] from comparison with precise baseline solution for Jan. 20, 2011. 


\subsection{Formation Control}

After acquisition of the 20-km wide formation in July 2010 (cf. [7] for details on the acquisition process) the commissioning of the formation maintenance function within the flight dynamics system was successfully performed. Within the period July 20-31 the formation control system was manually operated on a daily basis. Because of the achieved excellent control accuracy (i.e. $5 \mathrm{~m}$ RMS in cross-track (2D) and < $50 \mathrm{~m}$ RMS in along-track direction) and in order to safe precious cold-gas for a likely mission extension, it was decided to make use of the TDX hydrazine propulsion system to control the $20 \mathrm{~km}$ along-track formation within the remaining time of the mono-static commissioning phase (i.e. until begin of October). The hereby achieved performance (i.e. $<20 \mathrm{~m}$ RMS in cross-track and $<300 \mathrm{~m}$ RMS in along-track direction) was fully sufficient for the purpose of calibrating the TDX radar instrument for mono-static operation and surprisingly accurate considering the fact that only two formation maintenance maneuver pairs were performed per week in order to minimize the impact on radar operations.

Shortly after acquiring the tight formation (i.e. zero mean along-track separation) in October 2010 the ground formation control process became fully automated. Since then daily formation maintenance maneuvers with the cold-gas propulsion have been performed. Although the twomaneuver control concept primarily aims on the adjustment of the relative eccentricity vector, the precise control of the along-track separation is fundamental for the purpose of SAR cross-track interferometry, too. In order to comply with the tight mission schedule, a reliable and accurate formation control operation was demanded right from the beginning of the bi-static commissioning phase. The formation geometries flown at that time and within the first cycle of routine DEM acquisition are depicted in Fig. 5 making use of the Hill frame (as derived from Eq. 5). The radial / along-track separation is shown left and the cross-track separation is illustrated right.
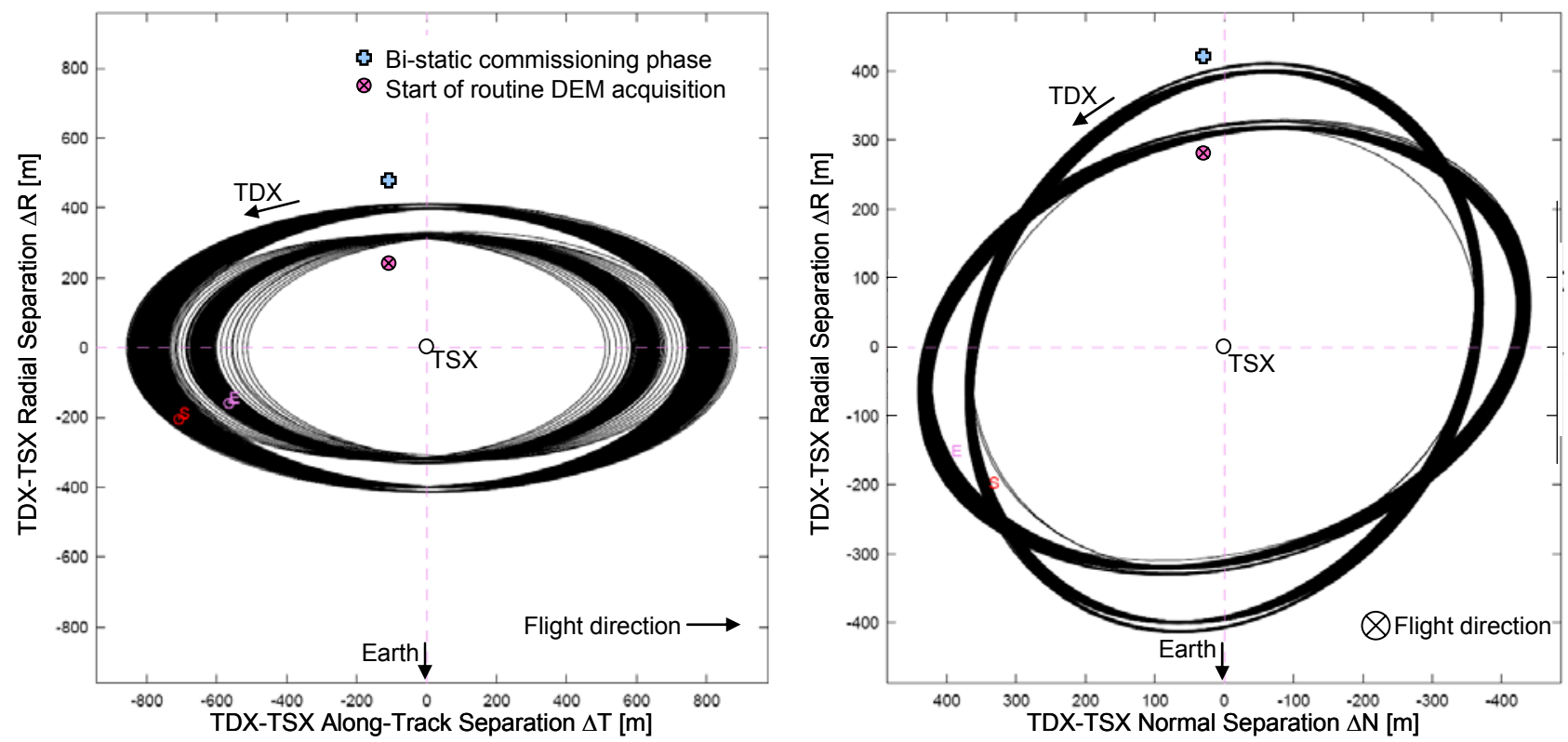

Figure 5. TDX-TSX relative motion in the Hill frame during the period from Oct. 16 to Dec. 31, 2010. Radial-/AlongTrack-Separation (left) and Radial-/Normal-Separation (right).

The following paragraphs concentrate on the control performance within the first 11-days cycle of the routine DEM acquisition phase performed in December 12-23, 2010. Figures 6 and 7 depict the evolution of relative orbital elements characterizing the out-of-plane and in-plane relative motion, respectively. The dashed red lines indicate upper and lower control limits, except for the relative semi-major axis $\Delta a$ where no limits apply. As discussed in Fig. 3 the target horizontal separation drifted from 443.0 to $426.2 \mathrm{~m}$ within 11 days. The drift was initiated by a small maneuver on Dec. 11 that installed a TDX-TSX relative inclination of $\Delta i=-9.6 \mathrm{E}-5$ deg (cf. Eq. 7, $a \delta \Delta i_{x}=-11.6 \mathrm{~m}$ ). 
Furthermore out-of-plane maneuvers were only necessary at the transition to the next 11-days cycle in order to reduce the drift (cf. Fig. 1).
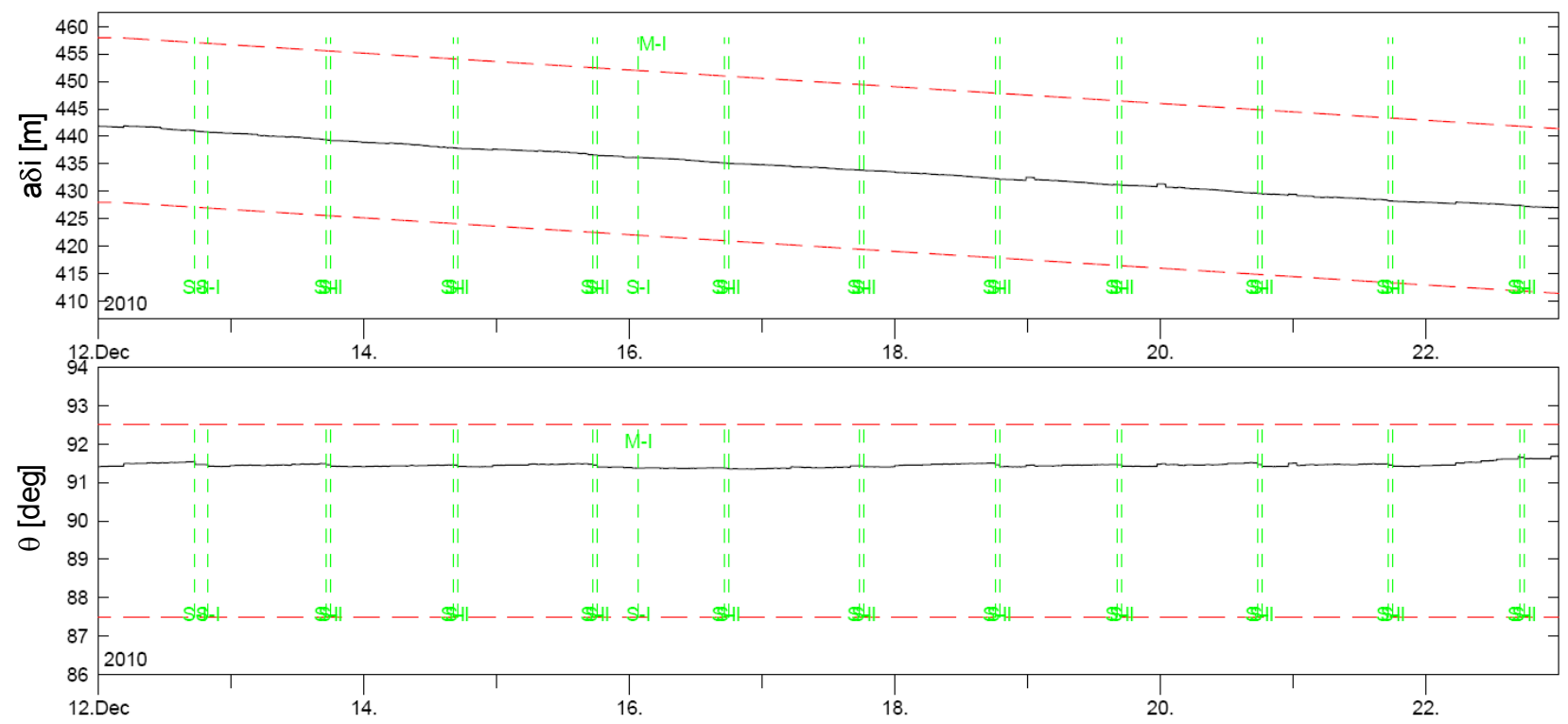

Figure 6. Relative orbital elements characterizing the out-of-plane motion for the period Dec.

12-23, 2010: magnitude of relative inclination vector $\mathbf{a} \delta i$ (top) and relative ascending node $\theta$ (bottom). Green verticals indicate common TSX/TDX (M-I) and individual TDX orbit maneuvers (S-I).

By means of Fig. 7 the in-plane control principle can be further illustrated. The first maneuver for relative eccentricity control occurs close to the violation of the lower limit of the relative argument of latitude $\varphi$ ( $2^{\text {nd }}$ diagram from top) and resets $\varphi$ close to its mean target value. As a result of this maneuver, $\Delta a$ can not remain close to zero. The direction and magnitude of the first maneuver are chosen in order to adjust the relative argument of latitude $a \Delta u$ within the short drift phase inbetween the two maneuvers. In case of large a $\Delta u$-offsets the nominal drift period of half an orbital period can be extended to 1.5 orbits or more to restore $a \Delta u$ in a faster way. An example can be found on Dec. 12, where the a $\Delta u$-deviation temporary exceeded $150 \mathrm{~m}$. The second maneuver completes the $\varphi$-adjustment to the upper control limit and brings $\Delta a$ close to zero. A small $\Delta a$ offset is often used to yield an average zero $a \delta \Delta u$ deviation. 

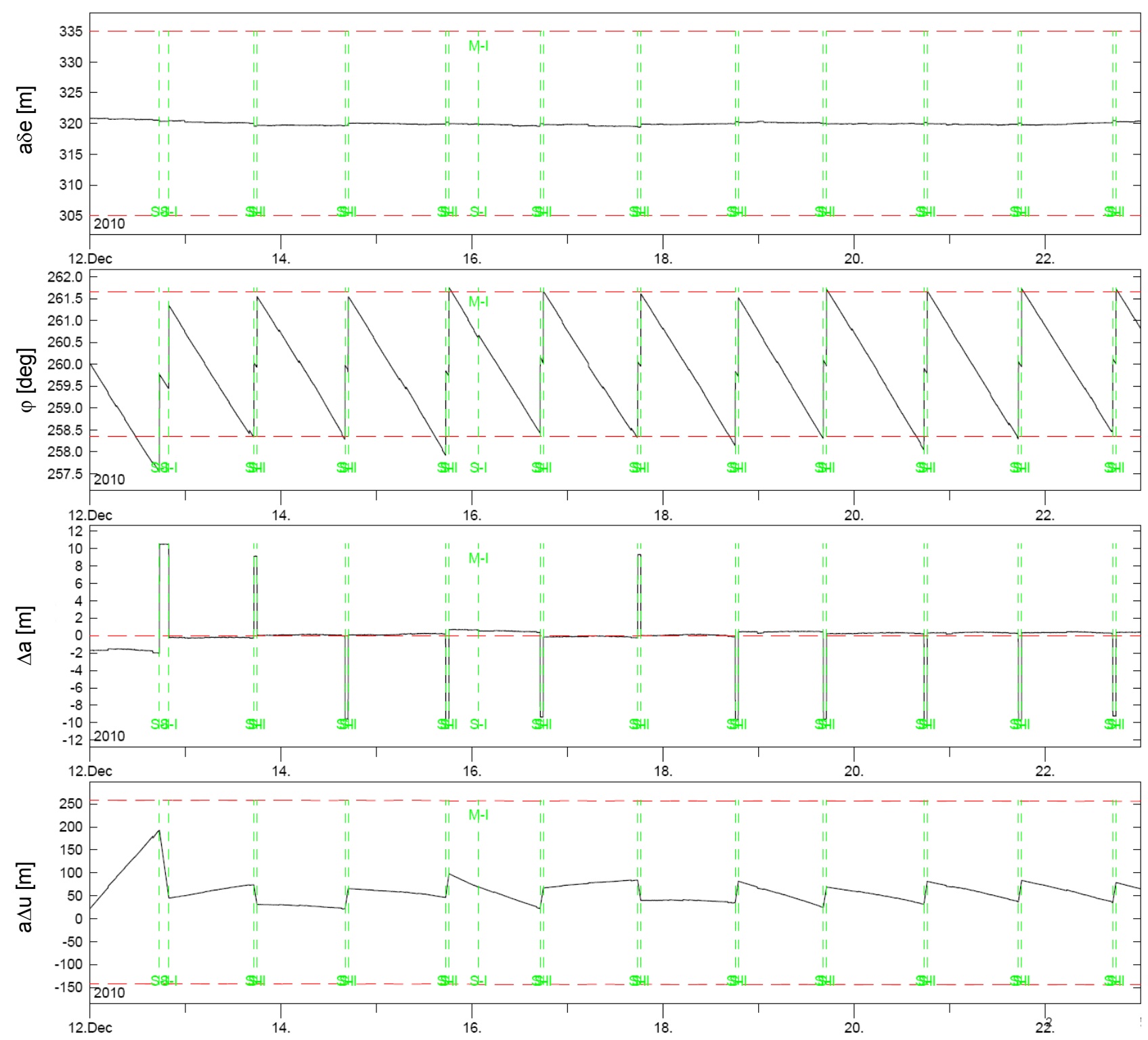

Figure 7. Relative orbital elements characterizing the in-plane motion for the period Dec. 12-

23, 2010. From top to bottom: magnitude of relative eccentricity vector a $\delta$, relative argument of perigee $\varphi$, relative semi-major axis $\Delta a$ and relative argument of latitude $a \Delta u$.

The expression of the relative orbital element deviations in the R-T-N system allows for a more precise analysis of the control performance in terms of SAR interferometry demands. The twodimensional cross-track error directly affects the height-of-ambiguity for cross-track interferometry. In Fig. 8 the derived along-track and cross-track errors are depicted. Because of the drifting eccentricity vector, a perfect alignment with the target elements is only achieved in the middle of a maneuver cycle where $\varphi$ crosses its nominal target value. An even better control performance can therefore only be achieved when decreasing the control cycle as foreseen with the TanDEM-X Autonomous Formation Flying System (TAFF, cf. [3] for more details), which will make use of nearly permanent availability of real-time relative navigation provided through an inter-satellite link (cf. Tab. 2) and therefore becomes independent from the ground-station contacts.

Tab. 4 summarizes required (right) and achieved relative control performance during the bi-static commissioning phase (left) and the first routine DEM acquisition cycle (middle). Note that the cross-track error is the magnitude of the $\Delta R-\Delta N$ error vector (cf. Eq. 6) and thus has a positive mean component while the individual mean errors are zero. The in-flight formation control performance is better than expected from the 10-month pre-launch simulation, where temporal violations of the control limits occurred especially in $a \Delta u$ [6]. As shown in Tab. 4 the achieved 
cross-track performance is a factor of 3 to 5 better than the required accuracy and a factor of 7 applies to the along-track accuracy. Both will be valuable when starting the combined processing (i.e. phase unwrapping) of DEM acquisitions from the first two years of formation flight.
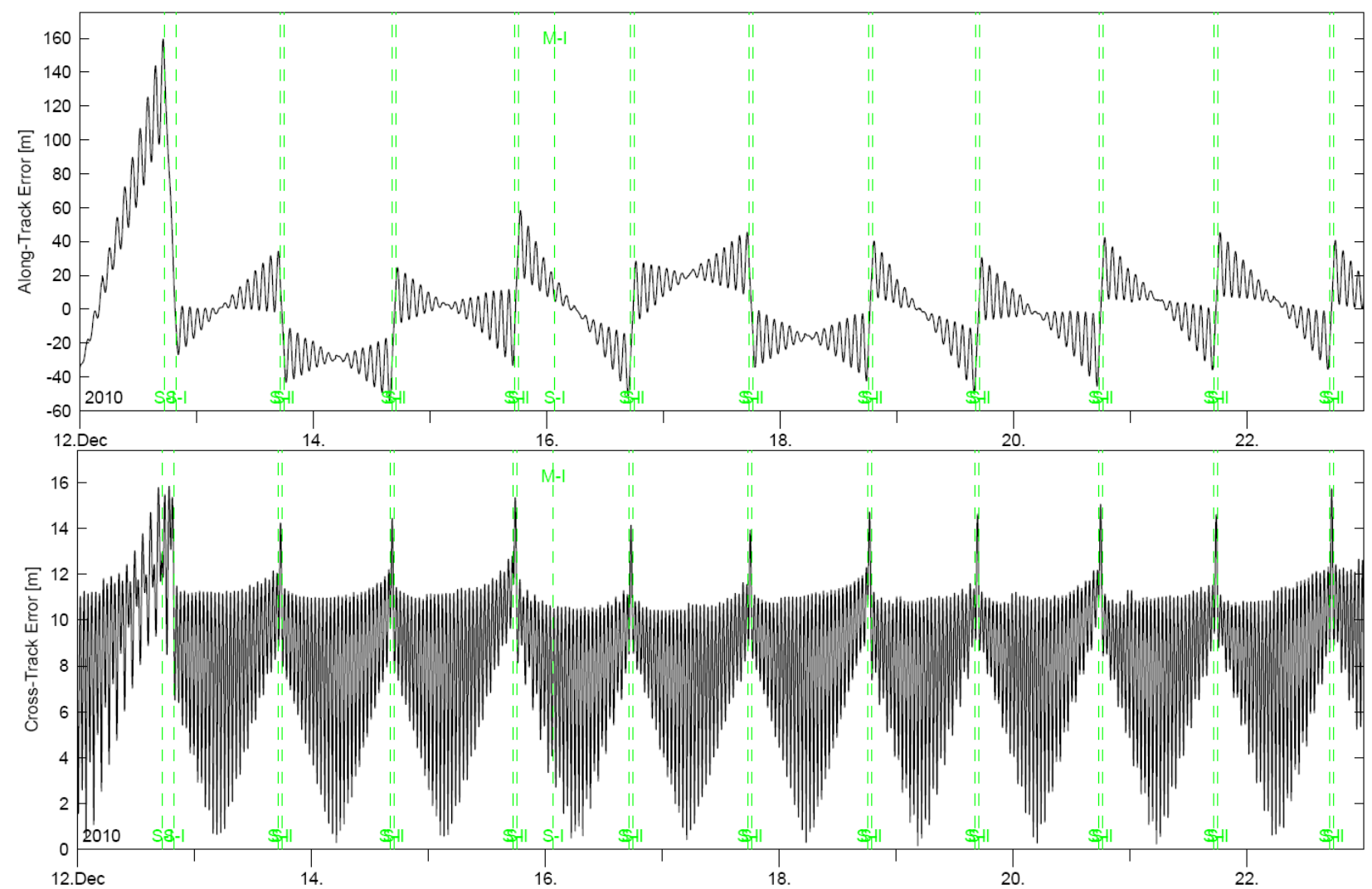

Figure 8. Along-track $\Delta T$ (top) and cross-track $\Delta C$ (bottom) deviations in the period Dec. 1223, 2010.

Table 4. Formation control accuracy achieved during bi-static commissioning phase and first routine DEM acquisition cycle with large horizontal drift and mission requirements (right).

\begin{tabular}{|l|r|r|r|r|r|r|r|}
\hline \multirow{2}{*}[\mathrm{m}]{} & \multicolumn{2}{|c|}{ Nov. $20-$ Dec. 1, 2010} & \multicolumn{2}{c|}{ Dec. 12-23, 2010 } & Required \\
\cline { 2 - 8 } & \multicolumn{1}{|c|}{ Mean } & \multicolumn{1}{c|}{ STD } & \multicolumn{1}{c|}{ RMS } & \multicolumn{1}{c|}{ Mean } & \multicolumn{1}{c|}{ STD } & \multicolumn{1}{c|}{ RMS } & \multicolumn{1}{c|}{ RMS } \\
\hline Radial, $\Delta \mathrm{R}$ & 0.0 & 5.2 & 5.2 & 0.0 & 4.5 & 4.5 & 20.0 \\
\hline Along-Track, $\Delta \mathrm{T}$ & -0.2 & 25.4 & 25.4 & 2.5 & 27.2 & 27.3 & 200.0 \\
\hline Normal, $\Delta \mathrm{N}$ & 0.0 & 0.9 & 0.9 & 0.0 & 7.8 & 7.8 & 20.0 \\
\hline Cross-Track, $\Delta \mathrm{C}$ (2D) & 4.2 & 3.2 & 5.3 & 8.6 & 2.7 & 9.0 & 28.3 \\
\hline
\end{tabular}

\section{Conclusion}

Within the first six months after TDX launch remarkable results could be achieved comprising the timely and fuel-efficient acquisition of the formation with TSX in July 2010, and the verification of safe and precise ground-in-the-loop formation control. So far neither contingencies nor space debris related issues were faced. The concept of collision avoidance for formation flying missions is discussed in [1] and was successfully simulated within a pre-launch software simulation [6].

The achieved relative orbit determination position accuracy is better than $50 \mathrm{~cm}$ in cross-track (2D, RMS) and about $1 \mathrm{~m}$ in along-track direction (RMS) and therefore well suited for the purpose of formation monitoring and control. After a short commissioning phase the GSOC formation control process has been fully automated. Daily in-plane maneuver pairs for execution on-board TDX are performed to compensate the natural eccentricity vector drift and to adjust the along-track 
separation while out-of-plane control is used to adjust the horizontal separation at less frequent control interval (typically 11 days). The achieved relative control accuracy is nominally $5 \mathrm{~m}$ (RMS) in cross-track direction and can be up to $10 \mathrm{~m}$ (RMS) during phases of large horizontal separation drifts as performed in the beginning of the DEM acquisition phase. The along-track control accuracy is better than $30 \mathrm{~m}$ (RMS). The control requirements of 28 / $200 \mathrm{~m}$ (RMS) in cross-track / along-track direction are clearly overachieved paving the way for the acquisition and processing of a global digital elevation model within the upcoming years of TSX-TDX formation flight.

\section{Acknowledgment}

The authors acknowledge the precious study and analysis work performed by Simone D'Amico on the concept of TSX-TDX formation control during the phases 0/A of the project.

The TanDEM-X project is partly funded by the German Federal Ministry for Economics and Technology (Förderkennzeichen $50 \mathrm{EE} \mathrm{0601)}$ and is realized in a public-private partnership (PPP) between German Aerospace Center (DLR) and Astrium GmbH.

\section{References}

[1] Aida S. and Kirschner M. (2011). Collision Risk Assessment and Operational Experiences for LEO Satellites at GSOC. 22nd ISSFD, Feb. 28 - Mar. 4, 2011, São José dos Campos, Brazil.

[2] Arbinger Ch., D’Amico S. and Eineder M. (2004). Precise Ground-In-the-Loop Orbit Control for Low Earth Observation Satellites. In Proc. 18th ISSFD, Munich, Germany.

[3] Ardaens J.S., D'Amico S., Ulrich D., Fischer D. (2008). TanDEM-X Autonomous Formation Flying System. 3rd International Symposium on Formation Flying, Missions and Technology, 23-25 April 2008, ESA/ESTEC, Noordwijk.

[4] D’Amico S. and Montenbruck O. (2006). Proximity Operations of Formation-Flying Spacecraft Using an Eccentricity/Inclination Vector Separation. Journal of Guidance, Control and Dynamics 29(3), 554-563.

[5] D'Amico S. (2010). Autonomous formation flying in low earth orbit. PhD thesis; Technical University of Delft. ISBN 978-90-5335-253-3.

[6] Kahle R. and Schlepp B. (2010). Extending the TerraSAR-X Flight Dynamics System for TanDEM-X. 4th International Conference on Astrodynamics Tools and Techniques, 3-6 May 2010, Madrid.

[7] Kahle R., Schlepp B. and Meissner F. (2011). TerraSAR-X / TanDEM-X Formation Acquisition - Analysis and Flight Results. 21st AAS/AIAA Space Flight Mechanics Meeting, New Orleans, Louisiana.

[8] Krieger, G., Moreira, A., Fiedler H., Hajnsek I., Werner M., Younis, M., Zink, M. (2010). TanDEM-X: A satellite formation for high-resolution SAR interferometry. IEEE Transaction on Geoscience and Remote Sensing: Vol 45, No. 11, Feb. 2010, pp. 3317 - 3341.

[9] Montenbruck O., Wermuth M. and Kahle R. (2010). GPS Based Relative Navigation for the TanDEM-X Mission - First Flight Results. ION-GNSS-2010 Conference, 21-24 Sep. 2010, Portland, Oregon. 